\title{
Worst-Case Wideband Radiated IEMI for Unshielded and Shielded Cables: A Statistical Analysis of the Main Influencing Parameters
}

\author{
Tao Liang, Giordano Spadacini, Senior Member, IEEE, Flavia Grassi, Senior Member, IEEE, and \\ Sergio A. Pignari, Fellow, IEEE
}

\begin{abstract}
This work deals with Intentional Electromagnetic Interference (IEMI) and presents statistical analysis of both common-mode (CM) and differential-mode (DM) disturbances induced in unshielded or shielded cables by a radiated, wideband, high-power electromagnetic (HPEM) pulse. The quantity of interest is the worst-case (i.e., maximum) peak in the time-domain waveform of the voltage that can be induced in a terminal interface of the harness by an HPEM pulse with specified bandwidth and energy density. Statistics is needed to account for the lack of knowledge on the direction of incidence and polarization of the impinging plane-wave field, which are treated as random variables. Consequently, the worst-case induced-voltage peak is characterized by a cumulative distribution function (cdf). The analysis puts into evidence the different properties of the cdf for unshielded and shielded cables, with reference to the influence exerted by the main system parameters, including the cable length, the height above ground, and terminal impedances.
\end{abstract}

Index Terms-High-power electromagnetic (HPEM) pulse, intentional electromagnetic interference (IEMI), statistical electromagnetic compatibility (EMC) models.

\section{INTRODUCTION}

$\mathrm{T}$ hreats of intentional electromagnetic interference (IEMI) are gaining increasing attention from researchers, who carried out extensive investigations in recent years, focused on standardization, susceptibility and hardening of equipment, as well as risk analysis and experimental verifications [1]-[5]. Grounds for this interest are in the growing concern for terrorism and other criminal events, along with the general awareness of the critical dependence on informationtechnology equipment in the ordinary running of todays' society.

IEMI is a quite general concept which encompasses various kinds of interference sources and coupling mechanisms: radiated and conducted, broadband and narrowband, high and low power, low-tech and high-tech sources [1]. Among the most challenging threats to equipment immunity, radiated wideband IEMI is characterized by the harmful radiation of high-power electromagnetic (HPEM) fields towards the victim system. Nowadays, high-tech disrupters like impulse-radiating

Manuscript received Dec. 14, 2018, revised Feb. 28, 2019 and April 1, 2019, accepted April 2, 2019.

This paper is for the Special Issue and is an expanded version from the 2018 IEEE Symposium in Singapore/Long Beach.

The authors are with Dipartimento di Elettronica, Informazione e Bioingegneria (DEIB), Politecnico di Milano, Milan, 20133 Italy. (email: \{tao.liang, giordano.spadacini, flavia.grassi, sergio.pignari\}@polimi.it) antennas can generate electric-field pulses characterized by peak values in the order of several $\mathrm{kV} / \mathrm{m}$, and spectrum density distributed over a large frequency range. For instance, subhyperband and hyperband waveforms spread the $90 \%$ of their energy in a band ratio (i.e., ratio of upper over lower frequency limit) greater than 3 and 10, respectively [6]. From the viewpoint of an EMC designer, interested in the susceptibility assessment, modeling and prediction of IEMI effects generated by such kind of disrupters is a challenging task due to uncertainty on the characteristics of the electromagnetic environment. Indeed, the most varied time-domain waveforms can be involved in HPEM attacks: single and repeated pulses, damped sinusoids, gaussian-modulated sinusoids, etc. Moreover, these terms are only ideal mathematical descriptors which cannot exhaustively account for the complexity of real time-domain waveforms and spectra generated by the specific technology implemented in a disrupter, as one can easily verify by surveying the literature (e.g., see [2, Fig. 1], [3, Fig. 2], [5, Fig. 5], [6]). Furthermore, the direction of incidence and the polarization of the electromagnetic field are inherently unknown in a real attack scenario, therefore risk analysis should conveniently rely on probabilistic techniques [4].

A combined worst-case/statistical approach for predicting the effects of wideband radiated HPEM pulses was firstly developed in [7]. In that paper, in order to provide safe-side assessment of system vulnerability, uncertain technological features of specific disrupting sources are left out of consideration, while the analysis is aimed at finding the worstcase spectrum and waveform of the HPEM pulse which maximizes the peak of the voltage induced across a target electric port in the system, for a given bandwidth and energy density, and for a specified direction of incidence and polarization of the electromagnetic field. It is, indeed, proved that this worst-case solution depends only on properties of the victim system [7]. Namely, the worst-case solution exhibits optimal energetic matching of the interfering field spectrum with the receiving properties of the victim system, which can be equivalently characterized as transmitting properties by reciprocity. Specifically, the system can be modeled as an unintentional radiator in any electromagnetic solver to evaluate the radiation pattern and input impedance through a single frequency-domain simulation. By virtue of the reciprocity 


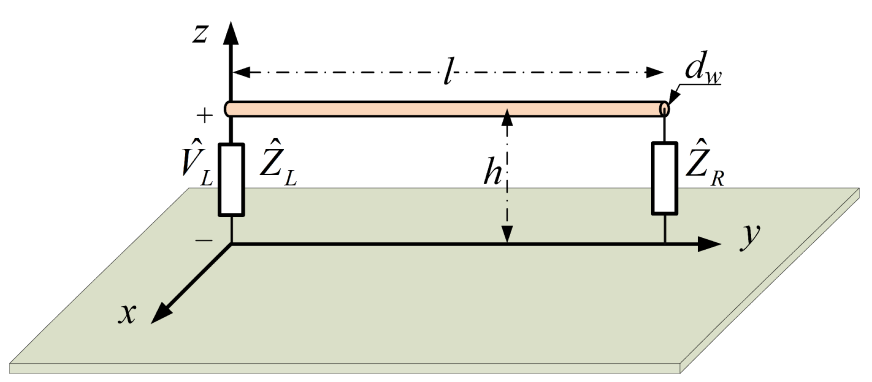

(a)

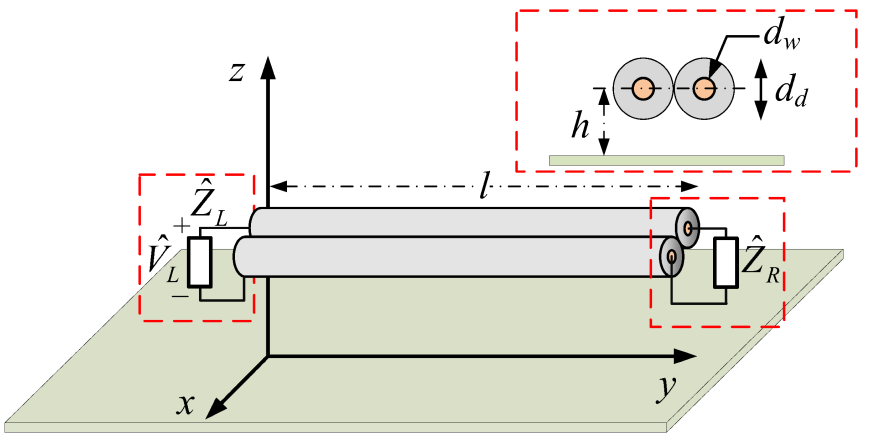

(b)

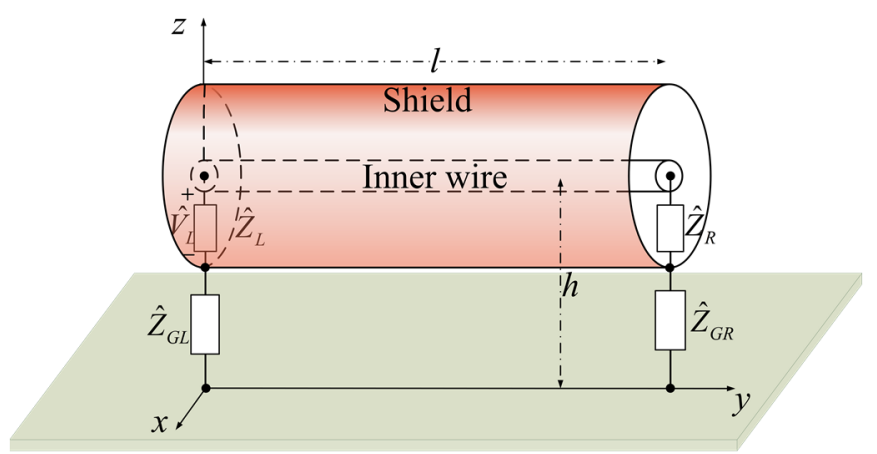

(c)

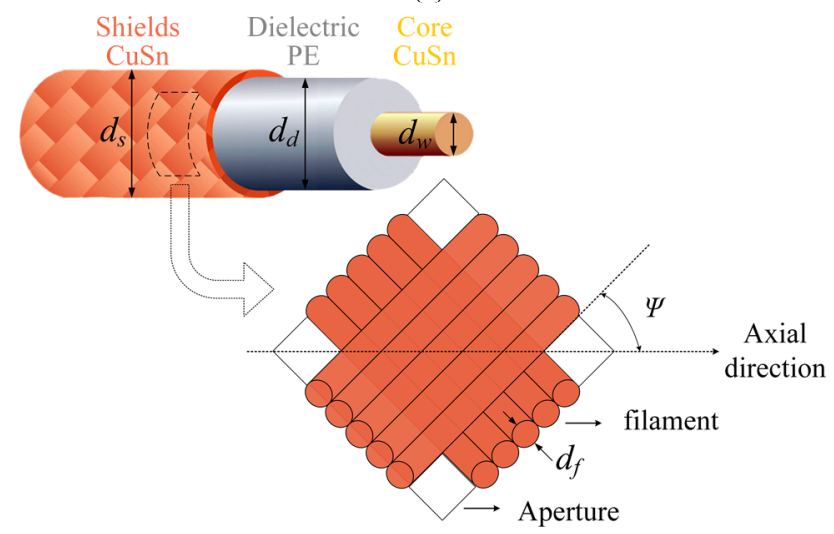

(d)

Fig. 1. The wiring structures under analysis: (a) Simplified CM representation of an unshielded harness as a single wire running above a metallic ground plane; (b) DL above a metallic ground; (c) a coaxial cable running above a metallic ground; (d) details of the coaxial cable.

theorem, this output is enough to compute the worst-case HPEM pulse waveform for any possible polar, azimuth, and polarization angles of the impinging electromagnetic field. Hence, by representing such angles as random variables (RVs), computationally efficient Monte Carlo simulations can be run

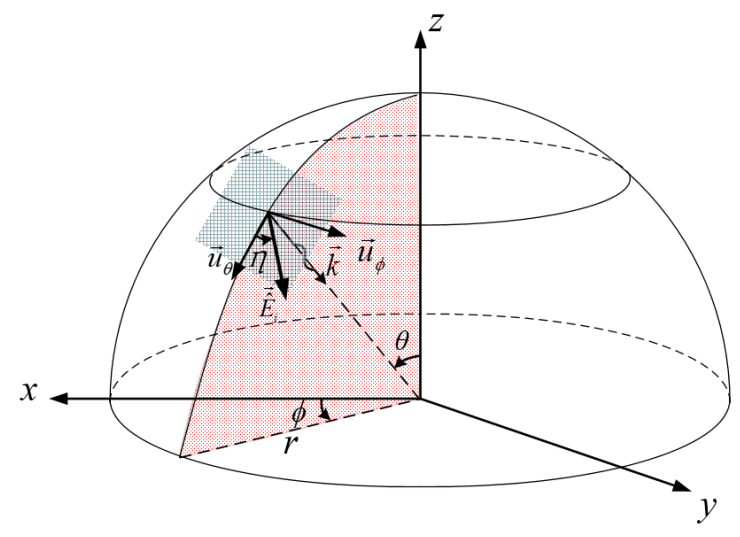

Fig. 2. Incidence and polarization angles of the plane-wave HPEM pulse defined in a spherical coordinate system.

for the computation of the cumulative distribution function (cdf) of the worst-case voltage peak.

In [9], such an approach was applied to one of the most relevant victim structures in IEMI studies, that is, an unshielded wiring harness running above ground, with specific focus on the dominant common-mode (CM) field-coupling mechanism. The obtained results were confirmed in [10] by an alternative analytical model, based on transmission-line (TL) theory in place of full-wave simulations. This paper reconsiders the analysis and conclusions presented in [9] and extends them to two other wiring configurations: a) differential-mode (DM) noise in an unshielded differential line (DL) and b) a shielded cable. Several simulations unveil the different role played by the main system parameters in shaping the cdf of the worst-case voltage peak. In particular, the influencing factors of interest include a) the cable length, b) the height above ground, c) the impedance of terminal equipment, d) grounding strategy. Moreover, similarities and differences exhibited by unshielded and shielded cables are discussed.

The paper is organized as follows. The victim structures under analysis and the main theoretical aspects of the method are summarized in Sec. II. Selected simulations and results are presented in Sec. III (for unshielded cables) and Sec. IV (for a shielded, coaxial cable) to corroborate the main findings. Specifically, it will be shown how the length of the harness plays a minor role in the statistics of the worst-case peak, whereas the main influencing factors result to be the height, terminal impedances, and the grounding strategies for shielded cables. Concluding remarks are drawn in Sec. V.

\section{RECIPROCITY-BASED COUPLING MODEL AND WORST- CASE HPEM PULSE WAVEFORMS}

\section{A. Structures under analysis}

As shown in Fig. 1(a)-(c), the three wiring structures of interest are basic representations of an unshielded harness, an unshielded differential line, and a shielded cable running above a metallic ground, respectively. These structures are intentionally simple and canonical in order to retain just the dominant aspects of coupling phenomena, since this paper is mainly targeted to derivation of general properties. Nevertheless, the proposed theory could be applied to detailed 
models of specific cable structures (e.g., bundles, twisted-wire pairs, etc.) at the expense of increased computational efforts in 3D simulations.

Specifically, the first structure under analysis represents a harness composed of an arbitrary number of wires and interconnected to terminal units. Since radiated susceptibility is characterized by a dominant $\mathrm{CM}$ coupling mechanism [8], in Fig. 1(a) the multi-wire harness is reduced to a single equivalent wire describing the propagation of $\mathrm{CM}$ voltages/currents [11]. The wire is bare and lossless, with diameter $d_{w}$ and length $l$, and runs at height $h$ above a perfectly conducting ground plane. CM impedances of left and right terminal units are $\hat{Z}_{L}$ and $\hat{Z}_{R}$, respectively. In this structure, DM noise is mainly caused by imbalance of terminal equipment and consequent conversion of the dominant CM noise, whose prediction is therefore of interest.

To analyze pure DM coupling, a two-wire DL floating above ground is defined in Fig. 1(b). Wires are lossless with length $l$, diameter $d_{w}$, and coated with dielectric material of relative electric permittivity $\varepsilon_{r}$ and external diameter $d_{d}$. Impedances $\hat{Z}_{L}$ and $\hat{Z}_{R}$ are connected to the left and right end, respectively.

The third wiring structure in Fig. 1(c) is a basic representative for the large category of shielded cables, that is, a coaxial cable of length $l$. The inner wire of diameter $d_{w}$ runs at height $h$ above ground. As shown in Fig. 1(d), the inner wire (tinned copper) is covered by dielectric material with relative electric permittivity $\varepsilon_{r}$, external diameter $d_{d}$, and a braided shield (tinned copper) of external diameter $d_{s}$ (dependent on shields parameters). The shield contains $m$ carriers, where, each carrier contains $n_{f}$ filaments of diameter $d_{f}$, and the weave angle is $\psi$. Terminal impedances $\hat{Z}_{L}$ and $\hat{Z}_{R}$ are connected between the inner wire and the shield, whereas the shield is grounded through impedances $\hat{Z}_{G L}$ and $\hat{Z}_{G R}$ at the left and right ends, respectively.

\section{B. Reciprocity-based coupling length}

Without loss of generality, the target variable of the proposed analysis is the voltage waveform $V_{\mathrm{L}}(t)$ (or, equivalently, its spectrum $\hat{V}_{L}$ in the frequency domain) induced across the left load impedance $\hat{Z}_{L}$ by the linearly-polarized electromagnetic wave shown in Fig. 2, whose incidence direction is identified by polar angle $\theta$ and azimuth angle $\phi$, and whose polarization is defined by angle $\eta$. The incident electric-field of the HPEM pulse is described by the time-domain waveform $E_{i}(t)$ or, equivalently, by its spectrum $\overrightarrow{\hat{E}}_{i}$ in the frequency domain.

The field-coupling model used in this paper takes advantage from a reciprocity-based approach, whose rationale is briefly summarized here, whereas the reader is referred to [7] for a complete theoretical derivation. First, the algorithm is based on the Lorentz reciprocity theorem, which establishes a noteworthy relationship between the radiated emission and the radiated susceptibility of a system. Specifically, for any linear system with an accessible electric port, the port voltage/current induced by field coupling can be reconstructed from reciprocal information about the far-field radiation pattern and the input

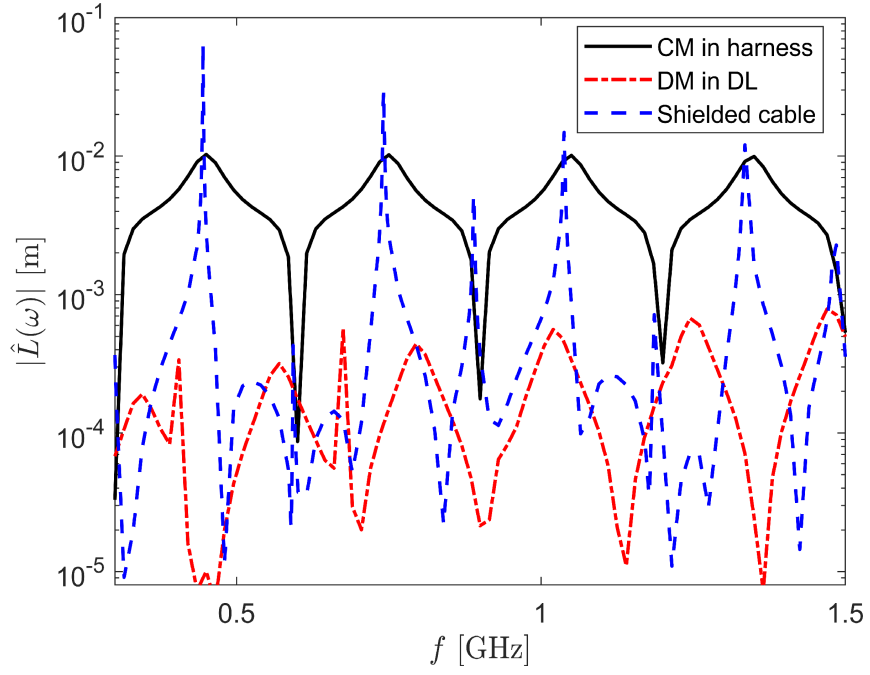

Fig. 3 Coupling length (magnitude) for the wiring structures presented in Fig. 1(a)-(c), respectively. See data in Sec. II.B.

impedance of the system itself, treated as an unintentional antenna.

In particular, each structure in Fig. 1(a)-(c) can be represented in any $3 \mathrm{D}$ electromagnetic solver and provided with a feeding port in place of the passive load impedance $\hat{Z}_{L}$. The structure is then solved in the frequency domain for: a) the farfield complex radiation pattern

$$
\overrightarrow{\hat{F}}=\hat{F}_{\theta} \cdot \vec{u}_{\theta}+\hat{F}_{\phi} \cdot \vec{u}_{\phi}
$$

decomposed in the two orthogonal components $\hat{F}_{\theta}, \hat{F}_{\phi}$, where $\vec{u}_{\theta}$ and $\vec{u}_{\phi}$ are versors in Fig. 2; b) the input impedance of the unintentional antenna $\hat{Z}_{a}$ at the feeding port. All these quantities are functions of the angular frequency $\omega$ in the bandwidth of interest.

In this paper, the structure in Fig. 1(a) was solved by a fullwave Finite-Integration Technique (FIT) [12], whereas the DL in Fig. 1(b) and the shielded cable in Fig. 1(c) were solved by a combined TL/Method of Moments (MoM) method [13]. The latter proved to be a computationally efficient solution for structures involving transverse-electromagnetic propagation mode in the bandwidth of interest [10]. For instance, in the coaxial-cable problem, the TL model was used to solve the interior domain (propagation of current in the two-conductor TL formed by the inner wire and the internal surface of the shield). Afterwards, the transfer impedance of the shield, multiplied by the current distribution computed in the interior domain, provided the distributed voltage acting as a CM source in the exterior domain [14]. The resulting current distribution flowing in the external surface of the shield, and its radiated field, are then computed by MoM.

Once the radiation pattern and input impedance are known from a single simulation, the load voltage induced by the electromagnetic field in Fig. 2 can be computed by

$$
\hat{V}_{L}(\omega)=\hat{L}(\omega) \cdot \hat{E}_{i}(\omega)
$$


where the transfer function $\hat{L}(\omega)$ represents a coupling length whose expression is cast by reciprocity in the form

$$
\hat{L}(\omega)=-\frac{\hat{Z}_{L}}{\hat{Z}_{a}+\hat{Z}_{L}} \frac{2 j \lambda_{0}}{Z_{0} \hat{I}_{a}}\left[\hat{F}_{\theta} \cos \eta+\hat{F}_{\phi} \sin \eta\right]
$$

where $j$ is the imaginary unit, $\lambda_{0}=c_{0} / f$ denotes the free-space wavelength $\left(c_{0} \approx 3 \times 10^{8} \mathrm{~m} / \mathrm{s}\right.$ is the speed of light in vacuum, $f$ is the frequency), $Z_{0} \approx 377 \Omega$ is the free-space wave impedance, and $\hat{I}_{a}$ is the arbitrary current used to feed the unintentional antenna at its port.

For the three wiring structures in Fig. 1, typical curves of coupling length versus frequency are plotted in Fig. 3. Common data are $d_{w}=0.9 \mathrm{~mm}, l=1 \mathrm{~m}, h=1 \mathrm{~cm}, \hat{Z}_{L}=\hat{Z}_{R}=50 \Omega$, $\theta=30^{\circ}, \varphi=0^{\circ}, \eta=45^{\circ}$. DL wires are coated with Polyethylene $\left(\varepsilon_{r}=2.3\right)$ and $d_{d}=2.95 \mathrm{~mm}$. The coaxial cable is a type RG-58 $\left(\varepsilon_{i}=2.3, d_{d}=2.95 \mathrm{~mm}, d_{s} \approx 3.50 \mathrm{~mm}, m=8, n_{f}=5, d_{f}=0.14 \mathrm{~mm}\right.$, $\psi=45^{\circ}$ ) with $\hat{Z}_{G L}=\hat{Z}_{G R}=1 \mathrm{~m} \Omega$. It is worth noting that the frequency responses of the coupling length for the unshielded and shielded configurations are qualitatively different. The CM in unshielded harness and the DM in a DL exhibit smooth behaviors, whereas the coaxial cable leads to low values (thanks to shielding) except for a limited number of very high, narrow peaks at resonant frequencies.

\section{Worst-case HPEM scenario}

In the proposed assessment method, the only quantities of the HPEM pulse object of specification are the bandwidth, which is limited in an interval $\left[\omega_{1}, \omega_{2}\right]$, the energy density $W$ expressed in $\mathrm{J} / \mathrm{m}^{2}$, and the wave angles $\theta, \phi, \eta$. Given these inputs, according to the solution of an optimization problem presented in [7], it is possible to find the worst-case spectrum of the HPEM pulse which maximizes the peak $V_{\mathrm{L}}\left(t_{0}\right)$ of the voltage induced at any desired time $t_{0}$, which results to be

$$
\hat{E}_{i}(\omega)=\sqrt{\frac{Z_{0} \pi W}{\int_{\omega_{1}}^{\omega_{2}}|\hat{L}(\omega)|^{2} d \omega}} \hat{L}(\omega)^{*} e^{-j \omega t_{0}}
$$

where the symbol * is used to denote complex conjugation. The optimized voltage peak is expressed by

$$
V_{L}\left(t_{0}\right)=\sqrt{\frac{Z_{0} W}{\pi} \int_{\omega_{1}}^{\omega_{2}}|\hat{L}(\omega)|^{2} d \omega}
$$

This solution describes a perfect energy matching between the spectrum of the incident field and the frequency-dependent receiving properties of the victim circuit. In practical terms, the available energy of the HPEM pulse is optimally distributed in the bandwidth so to favor those frequencies characterized by good receiving properties. Though very specific and perhaps unlikely to occur in a real attack scenario, this solution is valuable for a safe IEMI assessment, since it bounds any possible other response of the system under analysis [7].
By virtue of the Inverse Fourier Transformation (IFT), the worst-case electric-field waveform in the time domain is obtained by

$$
E_{i}(t)=\frac{1}{2 \pi} \int_{-\infty}^{+\infty} \hat{E}_{i}(\omega) e^{j \omega t} d \omega
$$

where, despite the theoretically infinite integration extremes, the integral can be computed within band limits only. Similarly, by substituting (4) in (2) and performing the IFT, the inducedvoltage waveform can be obtained both in frequency domain and in time domain.

In the framework of a Monte Carlo approach, the above analysis can be repeated for several values of the incidence and polarization angles of the plane-wave field (unknown in an IEMI attack). In this paper, these variables are actually treated as independent and uniformly (U) distributed RVs ranging in their widest definition intervals:

$$
\theta \sim \mathrm{U}\left[0^{\circ}, 90^{\circ}\right], \phi \sim \mathrm{U}\left[0^{\circ}, 360^{\circ}\right], \eta \sim \mathrm{U}\left[0^{\circ}, 360^{\circ}\right]
$$

By processing repeated-run simulations, the variability of the worst-case voltage peak (5) with the random incidence and polarization of the plane wave can be characterized through an empirical cdf. It is worth noting that this Monte Carlo approach is not computationally expensive, based on the same radiation pattern and the input impedance as long as the geometrical parameters of the structure are not changed.

\section{Statistics of the Worst-Case Voltage PeaK for CM AND DM IN AN UNSHIELDED HARNESS}

In this section, the impact of different geometrical/electrical parameters on the worst-case electric-field pulse, the worst-case voltage waveforms, and the cdf of the worst-case voltage peak is investigated through extensive Monte Carlo simulations. The following subsections present selected simulations and infer relevant properties.

Some input data and algorithmic aspects are in common with all the presented case studies. Namely, the HPEM pulse is bandlimited in the interval $[300 \mathrm{MHz}, 1.5 \mathrm{GHz}]$, and have energy density $W=1 \mathrm{~mJ} / \mathrm{m}^{2}$. For the computation of the radiation pattern, carried out through the full-wave solver [12], the space of variables $\theta$ and $\phi$ has been discretized with angular resolution of $1^{\circ}$, while the frequency has been linearly sampled with a step $\Delta f=30 \mathrm{MHz}$. The time instant $t_{0}$ when the voltage $V_{\mathrm{L}}\left(t_{0}\right)$ reaches the worst-case peak has been set (arbitrarily and for mere convenience) in the middle of the observation window according to discrete-IFT theory, that is, $t_{0}=1 /(2 \Delta f)=16.7 \mathrm{~ns}$.

\section{A. Impact of the harness length}

Without loss of generality, a specific set of incidence and polarization angles $\theta=30^{\circ}, \varphi=0^{\circ}, \eta=45^{\circ}$ is firstly considered to exemplify the impact of length on the worst-case electric field and the relevant induced voltage for the $\mathrm{CM}$ in an unshielded harness [Fig. 1(a)]. Fixed parameters are: $h=1 \mathrm{~cm}, d_{w}=2 \mathrm{~mm}$, 


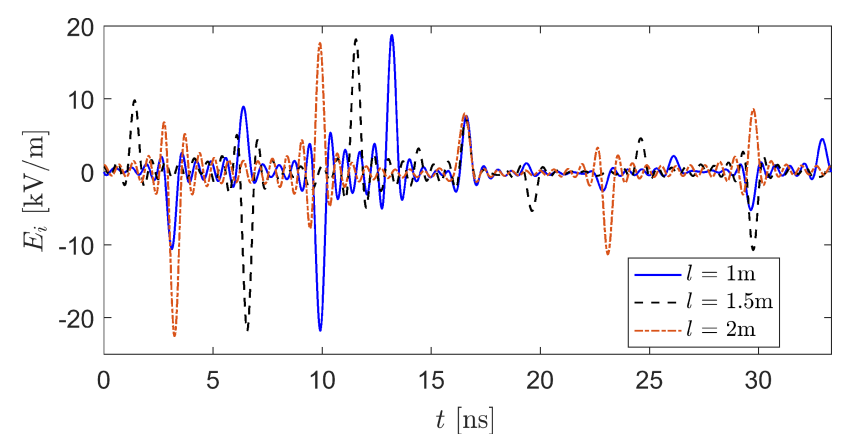

(a)

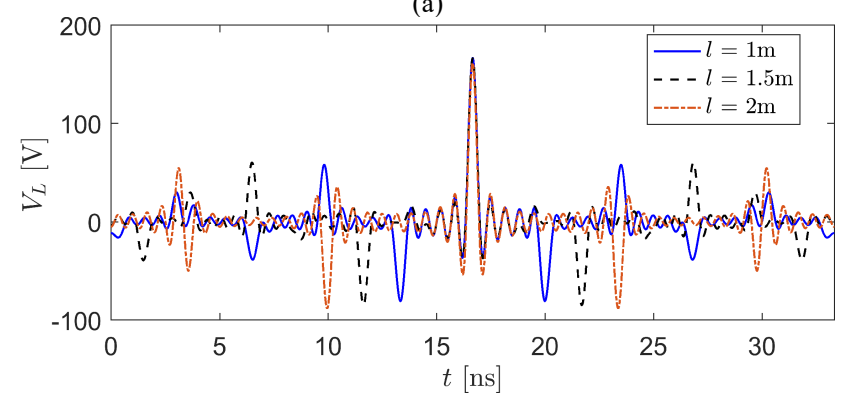

(b)

Fig. 4.: Impact of length on the waveform of (a) the worst-case incident field $E_{\mathrm{i}}$; (b) the worst-case induced voltage (CM in the unshielded harness).

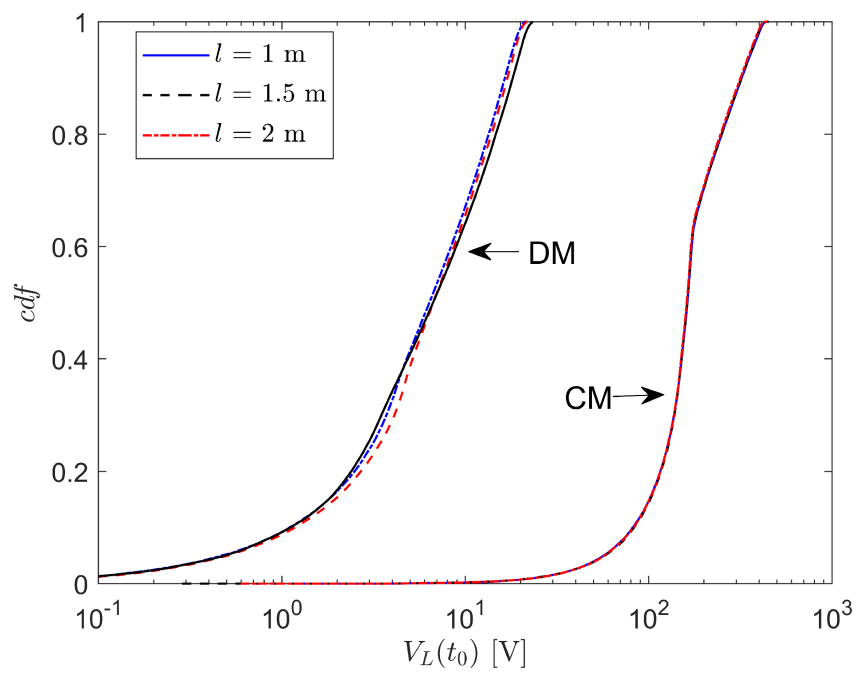

Fig. 5. Impact of the length on the cdf of the worst-case voltage peak $V_{\mathrm{L}}\left(t_{0}\right)(\mathrm{CM}$ in the unshielded harness and DM in the DL), estimated through Monte Carlo simulations ( $10^{5}$ random samples of incidence and polarization angles).

$\hat{Z}_{L}=50 \Omega, \hat{Z}_{R}=10 \Omega$, whereas three different harness lengths $l=1,1.5,2 \mathrm{~m}$ are considered.

Results are shown in Fig. 4(a)-(b) for the waveforms of the worst-case electric-field pulse and of the induced voltage, respectively. The worst-case waveforms present many repeated peaks and the ones of the voltage are symmetric with respect to time $t_{0}$ at which the maximum peak $V_{L}\left(t_{0}\right)$ is reached [7]. Repetitions of peaks in the voltage are consistent with many reflections occurring along the TL formed by the wire running above ground, whose characteristic impedance (estimated to be $Z_{C}=\sqrt{\mu_{0} / \varepsilon_{0}} \ln \left(4 h / d_{w}\right) / 2 \pi, Z_{C} \approx 179 \Omega$, where $\mu_{0}, \varepsilon_{0}$ are the free space permeability and permittivity, respectively) is unmatched with respect to the terminal resistive loads here considered. The incident electric-field shows similar repetitions which are purposely set by the worst-case solution (4) to optimize the induced voltage peak.

In Fig. 4(b), the maximum peak of the induced voltage $V_{\mathrm{L}}\left(t_{0}\right)$ does not significantly depend on the harness length (a close inspection of plotted curves shows a negligible variation between 161 and $166 \mathrm{~V}$ ). The cdf of the worst-case voltage peak was obtained by processing $10^{5}$ random sets of incidence and polarization angles, generated according to (7). Results are presented in Fig. 5 for CM in the unshielded harness and DM in the DL. One can appreciate that the cdfs corresponding to three different lengths are practically overlapped, thus confirming the above observation.

\section{B. Impact of terminal loads}

The port load $\hat{Z}_{L}$ (where the target voltage is induced) acts in a very simple fashion through a voltage divider (among the load impedance and the input impedance $\hat{Z}_{a}$ of the structure) visible at the beginning of equation (3). Owing to the voltage divider in (3), the higher is the load impedance, the higher is the induced voltage.

Conversely, the role played by $\hat{Z}_{R}$ at the opposite end of the wire is more complex. By resorting again to the analogy with TL theory, it is evident that this resistance determines the reflection coefficient at the right termination. To exemplify its impact, the $\mathrm{CM}$ and $\mathrm{DM}$ structures in Fig. 1(a)-(b) are characterized by $l=1 \mathrm{~m}, h=1 \mathrm{~cm}, d_{w}=2 \mathrm{~mm}, \hat{Z}_{L}=50 \Omega$ is considered. Different resistive values $\hat{Z}_{R}=10 \Omega, 180 \Omega$, $500 \Omega$, are specified. Fig. 6 reports the cdfs of the worst-case voltage peak obtained by processing $10^{5}$ random sets of incidence and polarization angles generated according to the statistical distributions in (7).

One can note that $\hat{Z}_{R}$ plays a fundamental and non-trivial role in shaping the cdf. The value of $180 \Omega$ is the closest to the $\mathrm{CM} / \mathrm{DM}$ characteristic impedance $Z_{\mathrm{C}}$ of the TLs $\left(Z_{\mathrm{C}} \approx 179 \Omega\right.$ for the $\mathrm{CM}$ in unshielded harness, $Z_{\mathrm{C}} \approx 120 \Omega$ for the DL). In this connection, one can note that the lack of impedance matching leads to higher quantiles of the distribution with respect to the nearly-matched case. For instance, the extreme value of $V_{\mathrm{L}}\left(t_{0}\right)$ for the $\mathrm{CM}$ is $228 \mathrm{~V}$ in case of matching, whereas its result almost doubled (449 V) for the case of low-resistance $\hat{Z}_{R}$.

\section{Impact of the height above ground}

The impact of the height is twofold. On the one hand, the CM characteristic impedance of the TL is modified (i.e., greater height implies greater $Z_{\mathrm{C}}$ ). In this respect, no role is played for the DM in Fig. 1(b), whereas a significant effect is expected for the CM in Fig. 1(a), depending on the reflection coefficient at the right terminal, as previously discussed. On the other hand, the height modifies the radiation pattern. In simple terms, increasing heights (within reasonable engineering limits for a harness) lead to improved patterns, that is, increased receiving properties. The superposition of both effects can be appreciated 


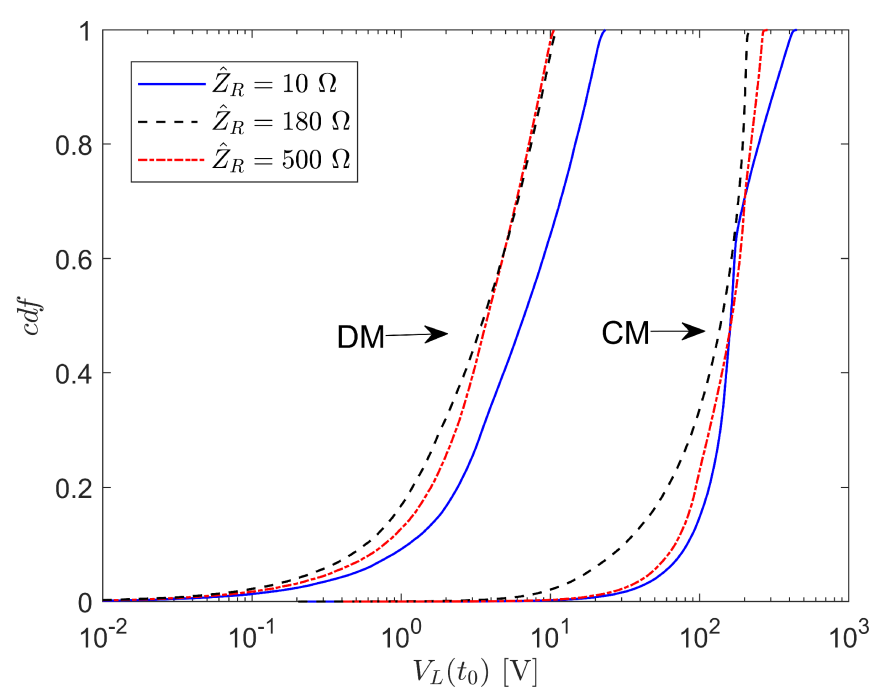

Fig. 6. Impact of $\hat{Z}_{R}$ on the cdf of the worst-case voltage peak $V_{\mathrm{L}}\left(t_{0}\right),(\mathrm{CM}$ in the unshielded harness and DM in the DL), estimated through Monte Carlo simulations $\left(10^{5}\right.$ random samples of incidence and polarization angles).

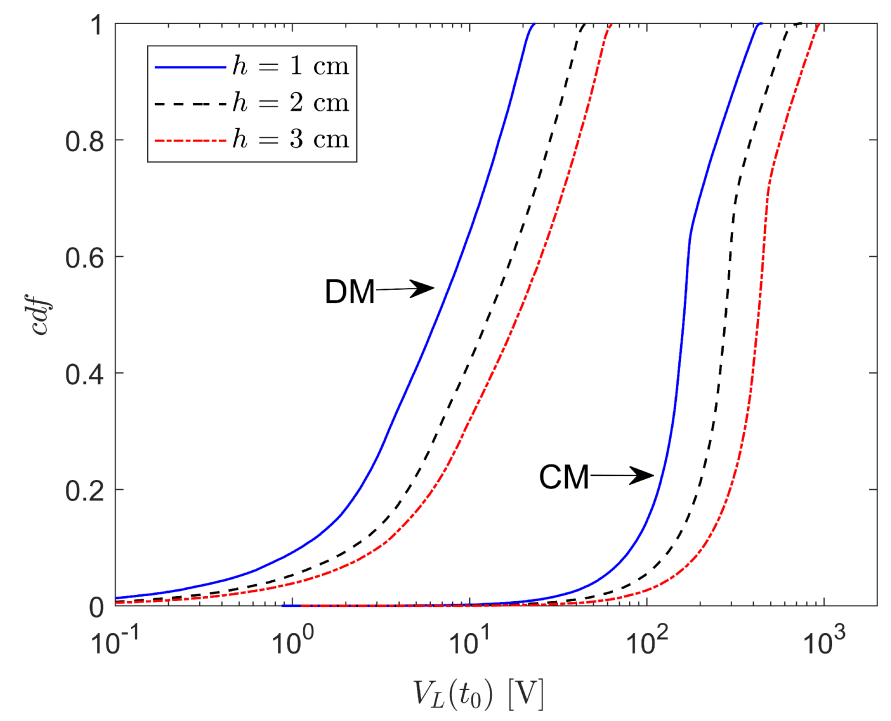

Fig. 7. Impact of height $h$ on the cdf of the worst-case voltage peak $V_{\mathrm{L}}\left(t_{0}\right),(\mathrm{CM}$ in the unshielded harness and DM in the DL), estimated through Monte Carlo simulations $\left(10^{5}\right.$ random samples of incidence and polarization angles).

in the cdf of the worst-case voltage peak (obtained by processing $10^{5}$ random sets of incidence and polarization angles) shown in Fig. 7 for structures characterized by $d_{\mathrm{w}}=2$ $\mathrm{mm}, l=1 \mathrm{~m}, \hat{Z}_{L}=50 \Omega, \hat{Z}_{R}=10 \Omega$, and three different heights $h=1,2,3 \mathrm{~cm}$. One can clearly appreciate that larger heights contribute to not only a generalized increase of quantiles, but also a change of shape of the cdfs.

For instance, for the $\mathrm{CM}$ in the unshielded harness, the median values ( 0.5 -quantile) of the peak voltages $V_{\mathrm{L}}\left(t_{0}\right)$ for $h=$ $1 \mathrm{~cm}, 3 \mathrm{~cm}, 5 \mathrm{~cm}$ are $162 \mathrm{~V}, 426 \mathrm{~V}$ and $596 \mathrm{~V}$ respectively. The extreme values (100\% percentile) are $450 \mathrm{~V}, 970 \mathrm{~V}$ and 1230 $\mathrm{V}$, respectively.

\section{Statistics of the Worst-Case Voltage Peak for A COAXIAL CABLE}

Going along the same path for the analysis of the unshielded harness, the impact of cable length, height and grounding impedances on the cdf of $V_{\mathrm{L}}\left(t_{0}\right)$ is discussed here for the coaxial cable in Fig. 1(c). The constraints of the HPEM field are the same, that is, bandwidth in [300 MHz, $1.5 \mathrm{GHz}]$, and energy density equal to $W=1 \mathrm{~mJ} / \mathrm{m}^{2}$.

The coaxial cable is an RG-58 with parameters defined in Fig. 1(c) and Section II.B. This cable has a characteristic impedance around $50 \Omega$ and its intended use is for matched loads, therefore, equal terminal impedances $\hat{Z}_{L}=\hat{Z}_{R}=50 \Omega$ are set. Conversely, different impedances $\hat{Z}_{G L}$ and $\hat{Z}_{G R}$ can be defined according to different grounding strategies, which play a significant role in the field-coupling response.

As previously exemplified in Fig. 3, the coupling length of a coaxial cable exhibits narrow resonances, which require a fine frequency sampling to accurately compute the integral in (5). These resonances are independent from wave angles and are approximately related to the cable length. By knowing the resonant frequencies in advance, uneven frequency sampling is implemented to assure computational efficiency. Specifically, the local frequency interval around each resonant frequency is sampled with a small step $\Delta f=1 \mathrm{MHz}$, whereas a larger step $\Delta f$ $=15 \mathrm{MHz}$ is used in the rest of the spectrum. The radiation pattern is computed through the combined TL/Full-wave approach described in Section II.B, and sampled with angular resolution of $1^{\circ}$ for both the angles $\theta$ and $\phi$.

A noticeable effect of these resonances is that the worst-case waveforms of the incident field and voltage result to be a combination of narrowband signals. Indeed, according to (4), the spectral content of the worst-case electric field is concentrated in the high resonance peaks of the coupling length occurring in the bandwidth of interest, which guarantees the best receiving potential from the structure (e.g., four/five frequencies in Fig. 3). Consequently, the worst-case waveforms are practically dominated by sums of narrowband signals with large duration and slow damping time. An example is shown in Fig. 8 for the following parameters, already considered in Fig. 3 to illustrate the coupling length: $\theta=30^{\circ}, \varphi=0^{\circ}, \eta=45^{\circ}, l=1 \mathrm{~m}$, $h=1 \mathrm{~cm}, d_{w}=0.9 \mathrm{~mm}, \hat{Z}_{G L}=\hat{Z}_{G R}=1 \mathrm{~m} \Omega$. The electric field and the induced voltage are plotted in Fig. 8(a), and Fig. 8(b), respectively.

The accuracy of the reciprocity-based computational method is verified in Fig.8(c), where the signal in Fig. 8(b) (in a short time windows for readability) is compared to a direct solution of the field coupling problem obtained by inputting the structure and the worst-case field in the full-wave solver [13].

In the remainder of this Section, the analysis will concentrate on the cdf of the worst-case voltage peak. 


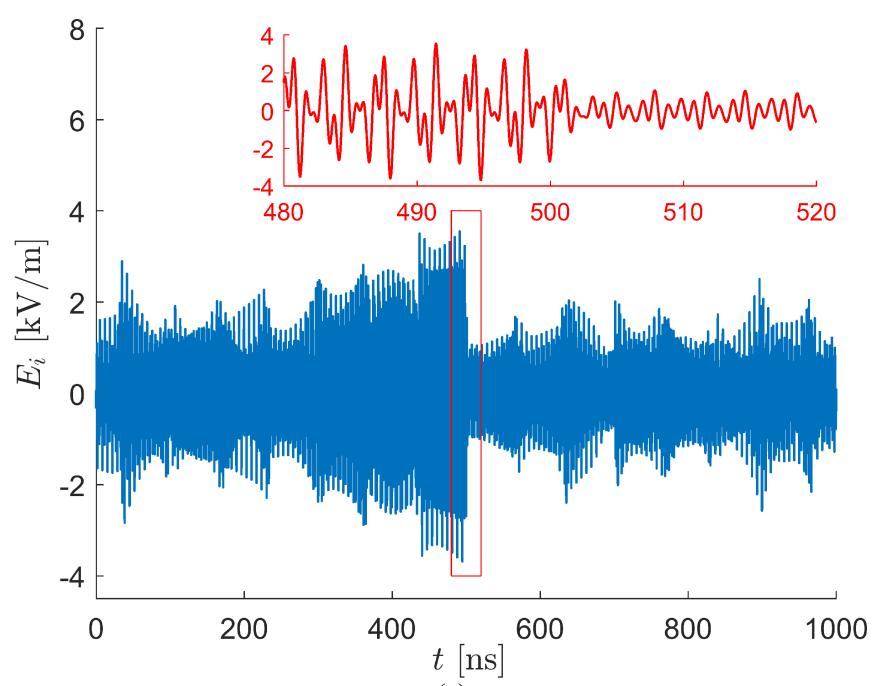

(a)
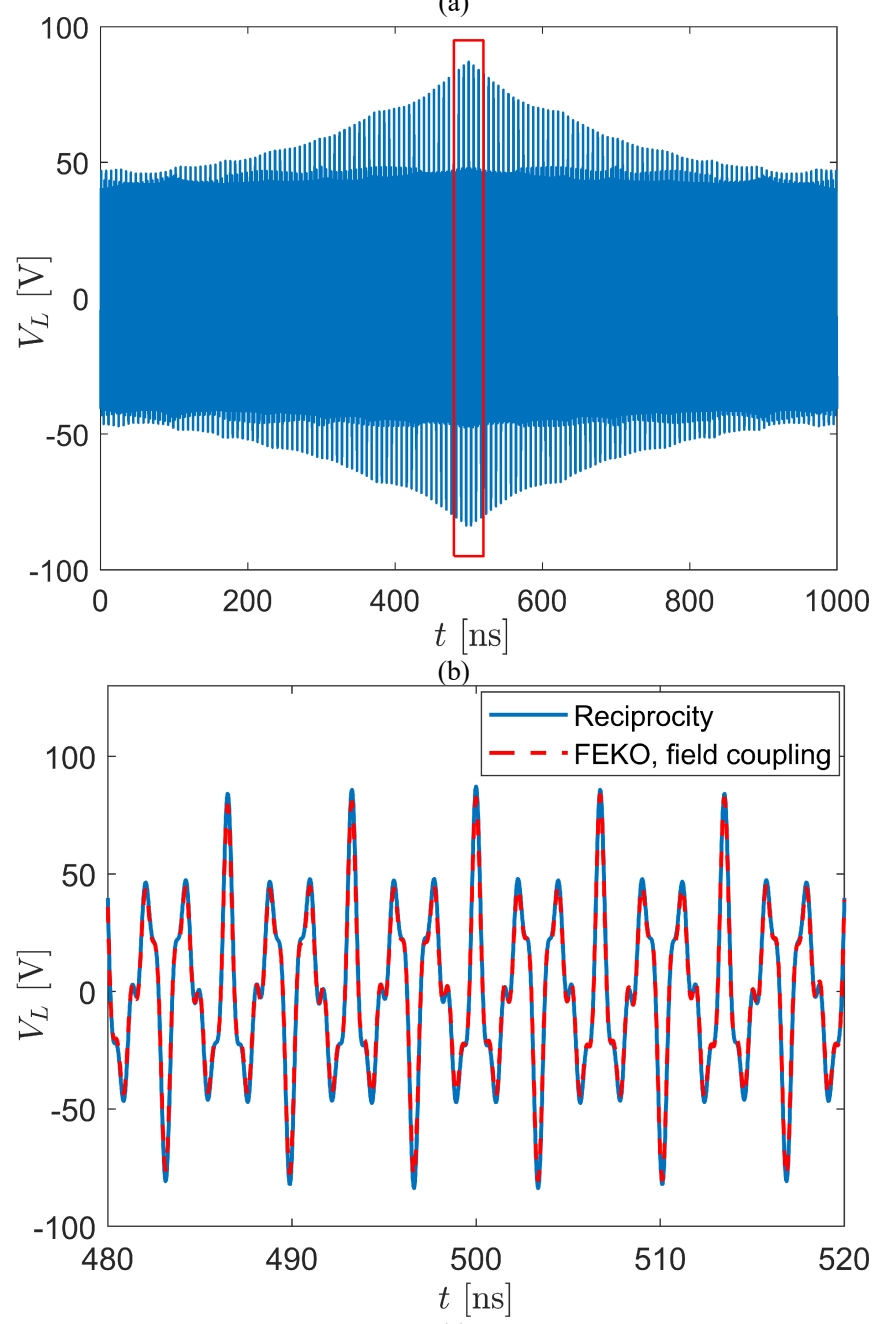

(c)

Fig. 8. Coaxial cable: (a) worst-case waveform of the incident field, the inset shows details in the short time windows; (b) worst-case waveform of the induced voltage. See data in Sec. IV. (c) Validation of the worst-case induced voltage in the rectangular window selected in (b).

\section{A. Impact of cable length}

Fig. 9 shows the cdfs of the worst-case induced-voltage peak $V_{\mathrm{L}}\left(t_{0}\right)$, computed through Monte Carlo simulations for $h=1 \mathrm{~cm}$, $\hat{Z}_{G L}=\hat{Z}_{G R}=1 \mathrm{~m} \Omega$, and three different cable lengths $(l=1 \mathrm{~m}, 1.5$ $\mathrm{m}, 2 \mathrm{~m})$. The inconsequentiality of length observed in Section II.A for the unshielded harness can be confirmed for the coaxial cable. Indeed, the cdfs in Fig. 9 are practically overlapped.

\section{B. Impact of the height above ground}

The cdfs of the worst-case induced-voltage peak $V_{\mathrm{L}}\left(t_{0}\right)$ computed through Monte Carlo simulation for $l=1 \mathrm{~m}$, $\hat{Z}_{G L}=\hat{Z}_{G R}=1 \mathrm{~m} \Omega$, and increasing height $(h=1 \mathrm{~cm}, 2 \mathrm{~cm}, 3 \mathrm{~cm})$ are plotted in Fig. 10.

One can notice that the impact of height is not very significant for the coaxial cable, unlike for the unshielded harness exemplified in Section II.C and Fig. 7. In fact, in Fig. 10 there is no evident correlation between a larger height and the increase of quantiles. Conversely, the upper extreme of $V_{\mathrm{L}}\left(t_{0}\right)$ slightly decreases from $250 \mathrm{~V}$ for $h=1 \mathrm{~cm}$ to $210 \mathrm{~V}$ for $h=3 \mathrm{~cm}$.

\section{Impact of grounding impedances}

Finally, the cdfs of $V_{\mathrm{L}}\left(t_{0}\right)$ for $l=1 \mathrm{~m}, h=1 \mathrm{~cm}$, and different grounding strategies are compared in Fig. 11. Specifically, four combinations of grounding impedances are considered: a) both ends grounded ( $\hat{Z}_{G L}=\hat{Z}_{G R}=1 \mathrm{~m} \Omega$ ); b) both ends floating ( $\hat{Z}_{G L}=\hat{Z}_{G R}=1 \mathrm{G} \Omega$ ); c) left end grounded, right end floating ( $\hat{Z}_{G L}=1 \mathrm{~m} \Omega, \hat{Z}_{G R}=1 \mathrm{G} \Omega$ ); d) left end floating, right end grounded ( $\hat{Z}_{G L}=1 \mathrm{G} \Omega, \hat{Z}_{G R}=1 \mathrm{~m} \Omega$ ).

One can appreciate the importance of grounding strategies in shaping the cdfs. Basically, the influence of grounding impedances is directly exerted on the current distribution induced on the external surface of the shield, variable with frequency, which in turn determines the distributed voltage sources in the interior domain of the shield through the transfer impedance, [14]. Therefore, the field-coupling mechanism is quite complex.

Actually, the cdfs in Fig. 11 do not univocally suggest a preferable grounding method for the reduction of interference. While one-end grounding leads to a cdf with the steepest slope, the floating case and the two-end grounding case demonstrate a slower growing trend. However, the latter reach the greatest extreme value. The common statistical estimates in Table 1 are of help to this comparison, namely, the mean, median (i.e., 0.5quantile), standard deviation (std) and maximum (max). On the basis of the mean and median values, the solution with both the shield terminals connected to ground appears the best one. However, for the reduction of the extreme value (generally of all quantiles above 0.75 ) the use of one-end grounding schemes may be considered.

TABLE 1. STATISTICAL ESTIMATES OF THE WORST-CASE VOLTAGE PEAK (CDFS IN FIG. 11)

\begin{tabular}{cccccc}
\hline \hline \multicolumn{2}{c}{ Grounding Condition } & \multicolumn{3}{c}{ Statistics of $V_{\mathrm{L}}\left(t_{0}\right),[\mathrm{V}]$} \\
\cline { 4 - 5 } Left-end & Right-end & Mean & Median & Std & Max \\
\hline Floating & Floating & 69.8 & 64.2 & 45.3 & 168.8 \\
Grounded & Floating & 83.8 & 88.7 & 28.5 & 132.3 \\
Floating & Grounded & 85.0 & 90.0 & 29.1 & 135.7 \\
Grounded & Grounded & 68.3 & 46.8 & 56.2 & 239.5 \\
\hline \hline
\end{tabular}




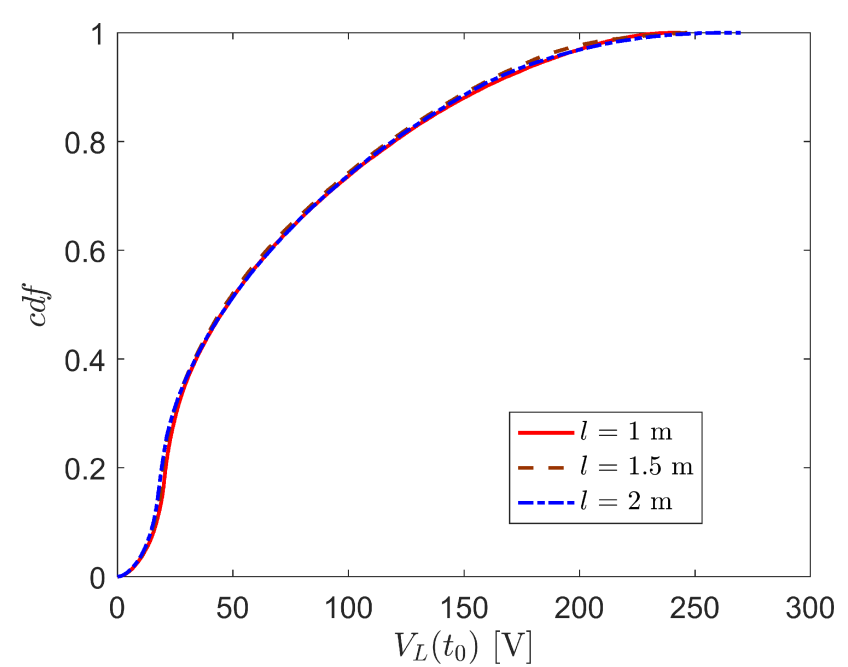

Fig. 9. Coaxial cable: Impact of the length on the cdf of the worst-case voltage peak $V_{\mathrm{L}}\left(t_{0}\right)$, estimated through Monte Carlo simulations $\left(10^{5}\right.$ random samples of incidence and polarization angles).

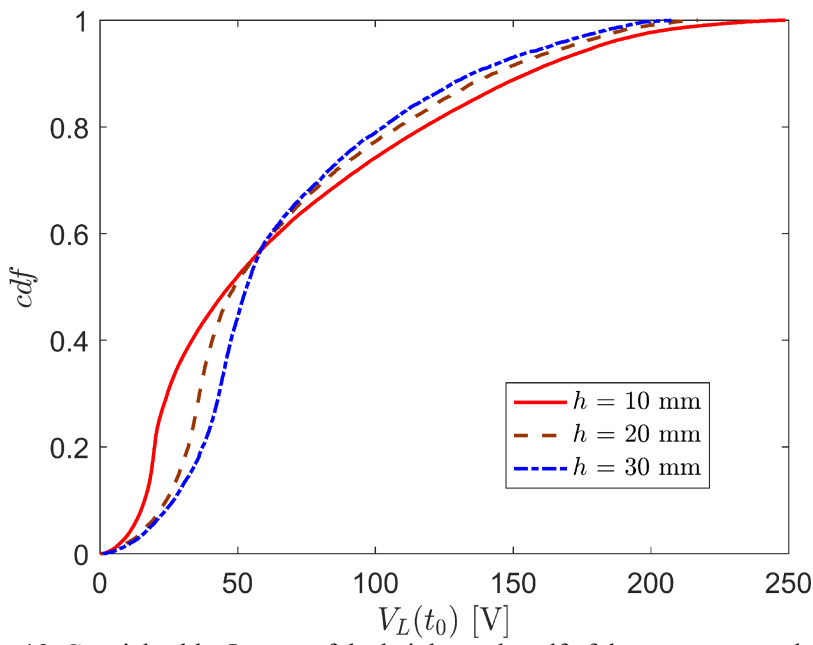

Fig. 10. Coaxial cable: Impact of the height on the cdf of the worst-case voltage peak $V_{\mathrm{L}}\left(t_{0}\right)$, estimated through Monte Carlo simulations $\left(10^{5}\right.$ random samples of incidence and polarization angles).

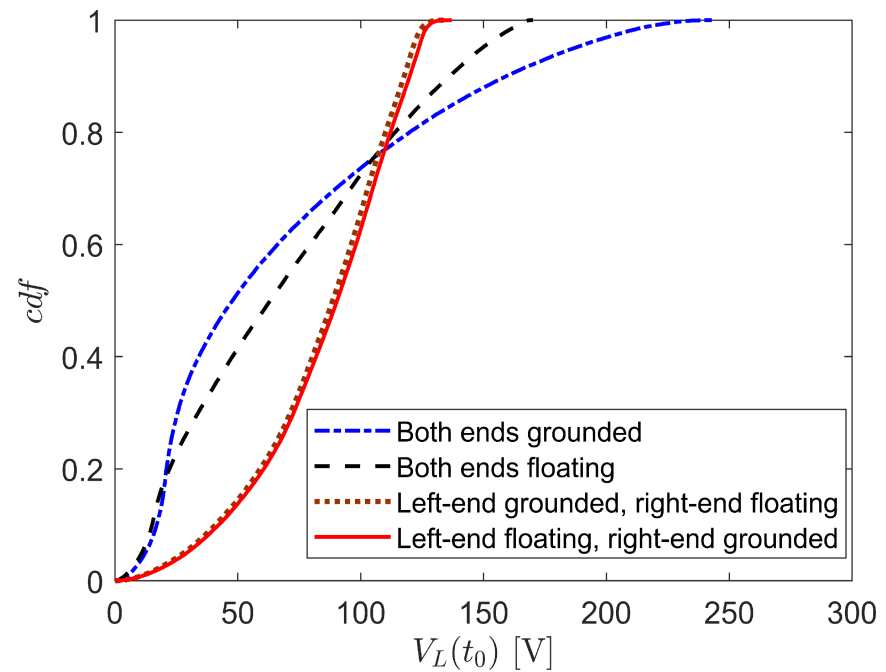

Fig. 11. Coaxial cable: Impact of grounding strategies on the cdf of the worstcase voltage peak $V_{\mathrm{L}}\left(t_{0}\right)$, estimated through Monte Carlo simulations $\left(10^{5}\right.$ random samples of incidence and polarization angles).

\section{CONCLUSIONS}

This works analyzed the impact of different influencing parameters on the statistics of the worst-case voltage peak induced across a terminal load by a wideband HPEM pulse having a specified bandwidth and energy density. Specifically, three victim structures were considered: a) an unshielded harness above ground, modeled for the dominant $\mathrm{CM}$ interference; b) an unshielded DL above ground, modeled for DM interference; c) a shielded (coaxial) cable above ground.

The proposed worst-case assessment optimizes the spectrum of the impinging electromagnetic field to match the receiving properties of the structure, characterized by its coupling length [7]. By the light of this approach, waveforms of field and induced voltage result to be quite different for the unshielded and shielded case. Specifically, since the coupling length for shielded cables exhibits a discrete number of high resonance peaks, the resulting worst-case waveforms are essentially combination of narrowband signals, of large duration and slow damping time. Conversely, the smooth coupling length of the unshielded cable leads to worst-case waveforms with wideband characteristics.

In all three structures of interest, the cable length turns out to play a negligible role on the statistics of the worst-case voltage peak. Extensive simulations (not reported for the sake of conciseness) demonstrated that this is true if the length is much greater than a critical length associated with the bandwidth of the HPEM pulse and the specific incidence direction [9]. This property is very interesting even from the computational viewpoint, since the full-wave solution of large structures involves considerable time and calculus resources. For the estimation of the statistical properties of IEMI effects, the solution could be carried out with reference to shorter harness lengths, without losing accuracy and completeness in the obtained results.

For the unshielded structures, the most important influencing factors proved to be a) the $\mathrm{CM}$ impedance connected at the opposite harness terminal (with respect to the load of interest in the analysis), and b) the height of the harness above ground. Both factors determine a significant impact on the reflection coefficients at TL terminals. In general, unmatched harness structures (with opposite impedance either higher or lower than the TL characteristic impedance) lead to worsening of IEMI effects in statistical terms, that is, to a generalized increase of the quantiles of the worst-case induced voltage peak. In addition, the height above ground inherently affects the radiation pattern, that is, the receiving properties of the wiring harness seen as an unintentional antenna. In general, the greater is the height above ground, the greater are the quantiles of the worst-case induced-voltage peak.

For the coaxial cable, the height plays a minor role. Conversely, the grounding impedances at shield terminals are of paramount importance. Specifically, the connection to ground of both the ends is an optimal solution to contain the mean and the median value of the worst-case inducted voltage peak. However, the use of single-end grounding is preferable to reduce the extreme value.

A number of extensions of the proposed results are desirable and still to be developed. A specific point concerns the definition of worst-case IEMI noise. While the proposed figure 
of merit was a global maximum in a voltage waveform, other signal norms (e.g., energy, duration, etc.) may be of interest, depending on the susceptibility mechanism of victim equipment [15].

\section{REFERENCES}

[1] W. A. Radasky, C. E. Baum, and M. W. Wik, "Introduction to the special issue on high-power electromagnetics (HPEM) and intentional electromagnetic interference (IEMI)," IEEE Trans. Electromagn. Compat., vol. 46, no. 3, pp. 314-321, Aug. 2004.

[2] D. Månnson, R. Thottappillil, T. Nilsoon, O. Lundén, M. Bäckström, "Susceptibility of civilian GPS receivers to electromagnetic radiation," IEEE Trans. Electromagn. Compat., vol. 50, no. 2, pp. 434-437, May 2008.

[3] F. Brauer, F. Sabath, and J. L. ter Haseborg, "Investigation of hardening measures for IT equipment against radiated and conducted IEMI," IEEE Trans. Electromagn. Compat., vol. 54, no. 5, pp. 1055-1065, Oct. 2012.

[4] E. Genender, H. Garbe, and F. Sabath, "Probabilistic risk analysis technique of intentional electromagnetic interference at system level," IEEE Trans. Electromagn. Compat., vol. 56, no. 1, pp. 200-207, Feb 2014

[5] N. Mora, I.D. Flintoft, L. Dawson, J. F. Dawson, F. Rachidi, M. Rubinstein, A. Marvin, P. Bertholet, and M. Nyffeler, "Experimental characterization of the response of an electrical and communication raceway to IEMI," IEEE Trans. Electromagn. Compat., vol. 58, no. 2, pp. 494-505, 2016.

[6] IEC 61000-2-13, Environment - High-power electromagnetic (HPEM) environments- radiated and conducted, 2005.

[7] G. Spadacini, T. Liang, F. Grassi, and S. A. Pignari, "Worst case and statistics of waveforms involved in wideband intentional electromagnetic attacks," IEEE Trans. Electromagn. Compat., vol. 60, no. 5, pp. 14361444, Oct. 2018.

[8] G. Spadacini, F. Grassi, and S. A. Pignari, "Field-to-wire coupling model for the common mode in random bundles of twisted-wire pairs," IEEE Trans. Electromagn. Compat., vol. 57, no. 5, pp. 1246-1254, Oct. 2015.

[9] T. Liang, G. Spadacini, F. Grassi, and S. A. Pignari, "Coupling of wideband radiated IEMI to wiring harness: a statistical analysis of the main influencing parameters," in Proc. IEEE Symp. on Electromagnetic Compatibility, Signal and Power Integrity, Long Beach, CA, USA, July 30-Aug. 3, 2018, pp. 357-362.

[10] T. Liang, G. Spadacini, F. Grassi, and S. A. Pignari, "Coupling of wideband radiated IEMI to cables above ground," IEEE Trans. Electromagn. Compat., pp. 1-9, 2018. (early assess article)

[11] G. Andrieu, L. Koné, F. Bocquet, B. Démoulin, and J.-P. Parmantier, "Multiconductor reduction technique for modeling common-mode currents on cable bundles at high frequency for automotive applications," IEEE Trans. Electromagn. Compat., vol. 50, pp. 175-184, 2008.

[12] CST Microwave Studio. (2016): www.cst.com

[13] User Manual for FEKO 2018. (2018): https://altairhyperworks.com/

[14] T. Kley, "Electromagnetic coupling to and from shielded cablesoptimizing factors," IEEE Trans. Electromagn. Compat., vol. 34, pp. 3946, 1992.

[15] D. V. Giri, High-Power Electromagnetic Radiators: Nonlethal Weapons and Other Applications. Cambridge, MA, USA: Harvard Univ. Press, 2004.

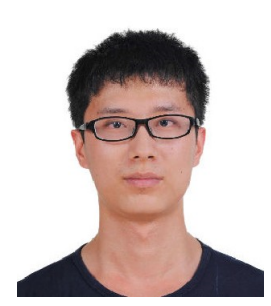

Tao Liang received the B.Sc. in electrical engineering from Xi'an Jiaotong University, Shaanxi, China, in 2013, received double M.Sc. degree in electrical engineering from both Xi'an Jiaotong University and Politecnico di Milano, Milan, Italy in 2016. He is currently working towards the Ph.D. degree in electrical engineering at Politecnico di Milano. His research interests include electromagnetic compatibility modeling and testing techniques.

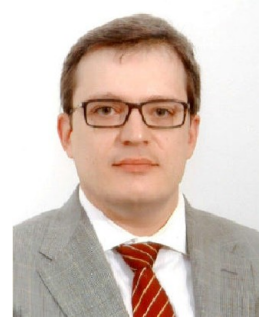

Giordano Spadacini (M'07-SM'16) received the Laurea (M.Sc.) and Ph.D. degrees in electrical engineering in 2001 and 2005, respectively, from Politecnico di Milano, Italy, where he is currently an Associate Professor with the Dept. of Electronics, Information and Bioengineering. His research interests include statistical models for the characterization of interference effects, distributed parameter circuit modeling, experimental procedures and setups for EMC testing, and EMC in aerospace and railway systems.

Dr. Spadacini has been Distinguished Reviewer of the IEEE EMC Transactions from 2014 to 2017. He is a recipient of the 2005 EMC Transactions Prize Paper Award, the 2016 Richard B. Schulz Best Transactions Paper Award, two Best Symposium Paper Awards from the 2015 Asia-Pacific Int. Symp. on EMC (APEMC) and the 2018 Joint IEEE EMC \& APEMC Symposium.

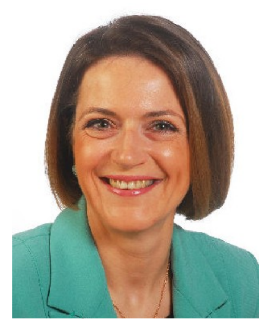

Flavia Grassi (M'07-SM'13) received the Laurea (M.Sc.) and Ph.D. degrees in electrical engineering from Politecnico di Milano, Italy, in 2002 and 2006, respectively, where she is currently an Associate Professor with the Dept. of Electronics, Information and Bioengineering. From 2008 to 2009, she was with the European Space Agency (ESA), The Netherlands, as a Research Fellow. Her research interests include distributed parameter circuit modeling, statistical techniques, characterization of measurement setups for EMC testing, and powerline communications.

Dr. Grassi was awarded the URSI Young Scientist Award in 2008, and the IEEE Young Scientist Award at the 2016 Asia-Pacific Int. Symp. on EMC (APEMC). She was a recipient of the IEEE EMC Society 2016 Transactions Prize Paper Award, and of the Best Symposium Paper Award from the 2015 APEMC and from the 2018 Joint IEEE EMC \& APEMC Symposium.

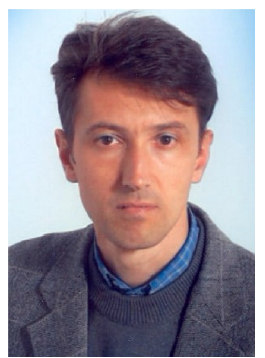

Sergio Amedeo Pignari (M'01-SM'07-F'12) received the Laurea (M.Sc.) and Ph.D. degrees in electronic engineering from Politecnico di Torino, Italy, in 1988 and 1993, respectively.

From 1991 to 1998, he was an Assistant Professor with the Dept. of Electronics, Politecnico di Torino, Turin, Italy. In 1998, he joined Politecnico di Milano, Milan, Italy, where he is currently a Full Professor with the Dept. of Electronics, Information, and Bioengineering, and Chair of the B.Sc. and M.Sc. Study Programs in Electrical Engineering. His research interests are in the field of EMC and include field-to-wire coupling and crosstalk, conducted immunity and emissions in multi-wire structures, statistical techniques for EMC, and experimental procedures and setups for EMC testing. His research activity is mainly related to Aerospace, Automotive, Energy, and Railway industry sectors.

Dr. Pignari is a recipient of the 2005 and 2016 IEEE EMC Society Transactions Prize Paper Award, and a 2011 IEEE EMC Society Technical Achievement Award. He is currently serving as an Associate Editor of the IEEE Transactions on EMC. From 2010 to 2015 he served as the IEEE EMC Society Chapter Coordinator. He has been Technical Program Chair of the ESA Workshop on Aerospace EMC in 2009, 2012, and 2016, and a Member of the Technical Program Committee of the Asia Pacific EMC Week since 2010. 